\title{
Bilateral breast cancer with a unilateral carcinoma within a fibroadenoma: A case report
}

\author{
HONGMEI ZHENG ${ }^{1}$, JIAN CHEN ${ }^{2}, \mathrm{XINHONG} \mathrm{WU}^{1}$, LITING JIN ${ }^{1}$ and CHUBO QI ${ }^{3}$ \\ Departments of ${ }^{1}$ Breast Surgery, ${ }^{2}$ Head and Neck Surgery and ${ }^{3}$ Pathology, \\ Hubei Cancer Hospital, Wuhan, Hubei 430079, P.R. China
}

Received August 22, 2014; Accepted May 7, 2015

DOI: $10.3892 / \mathrm{ol} .2015 .3420$

\begin{abstract}
Fibroadenomas are a type of benign tumor that occur in young women below the age of 35 years old. The tumors are the second most common type of tumor after fibrocystic disease. The chance of carcinoma arising in a fibroadenoma is extremely low. To date, $<130$ such cases have been reported. Previous studies have reported that fibroadenomas can evolve into a number of different types of malignancy. The present study is the first to describe a case of bilateral primary breast cancer with a unilateral invasive ductal carcinoma within a fibroadenoma. The current study presents a case of a 48-year-old female who presented with 2 stiff lumps on bilateral breasts, diagnosed as bilateral breast carcinoma, with a unilateral invasive ductal carcinoma within a fibroadenoma in the right breast. The patient underwent a bilateral mastectomy and subsequently received 4 cycles of chemotherapy (epirubicin, $60 \mathrm{mg} / \mathrm{m}^{2}$ and cyclophosphamide, $600 \mathrm{mg} / \mathrm{m}^{2}$ ) every 21 days, followed by 4 cycles of docetaxel chemotherapy $\left(100 \mathrm{mg} / \mathrm{m}^{2}\right)$ every 21 days. The patient then received maintenance endocrine therapy (tamoxifen, $20 \mathrm{mg}$, twice daily) for 19 months. The patient was followed up every 3 months, and at the last follow-up examination in May 2015, the patient exhibited no signs of recurrence.
\end{abstract}

\section{Introduction}

Breast cancer is the leading cause of cancer-associated mortality among females worldwide, accounting for $\sim 1.7$ million cases and 521,900 mortalities in 2012 (1), and invasive ductal carcinoma is the most common pathological type (1). The relative five-year survival rate of invasive ductal carcinoma ranges from $12 \%$ in parts of Africa to $90 \%$ in the United States, Australia and Canada (2). At Hubei Cancer Hospital (Wuhan, China), the patients with breast cancer often present with a painless mass

Correspondence to: Professor Xinhong Wu, Department of Breast Surgery, Hubei Cancer Hospital, 116 Zhuo Dao Quan South Road, Wuhan, Hubei 430079, P.R. China

E-mail: wuxinhong_9@163.com

Key words: breast cancer, fibroadenoma in the breast and the most commonly used examination method is mammary ultrasonography. Currently, the therapeutic methods used for breast cancer include surgery, chemotherapy, radiotherapy, endocrine therapy and targeted therapy (3). Fibroadenomas are a type of benign tumor that occur in younger women below the age of 35 years old. The tumors are the second most common type of tumor after fibrocystic disease (4). The chance of a carcinoma arising in a fibroadenoma is $0.1-0.3 \%$ (5). Previous studies have reported that fibroadenomas can evolve into different types of malignancy, such as lobular intraepithelial neoplasia, lobular carcinoma in situ, malignant phyllodes tumors and microinvasive lobular carcinoma (4,6-9). However, to the best of our knowledge, the present study is the first to report a case of bilateral primary breast cancer with a unilateral invasive ductal carcinoma within a fibroadenoma. Lumpectomy is the most frequent treatment for fibroadenoma, with a good prognosis in most cases (10-12). The current case of breast carcinoma within a fibroadenoma is indeed a rare mammary tumor. Written informed consent was obtained from the patient for the present study.

\section{Case report}

A 48-year-old female presented with a $3.0-\mathrm{cm}$ palpable, well-circumstanced, rough, stiff lump in the upper outer quadrant of the left breast, and a 2.0-cm palpable, smooth and mobile lump in the upper inner quadrant of the right breast. Clinical examination for other palpable masses in the breasts was negative, and there was no axillary lymphadenopathy. Breast ultrasonography revealed a poorly-defined palpable solid lesion $(3.0 \mathrm{~cm}$ in diameter) in the left breast with the sonographic features of a malignant tumor, and a well-defined solid lesion $(2.0 \mathrm{~cm}$ in diameter) with the sonographic features of a hypoechoic mass and calcification in the right breast (Fig. 1). The patient underwent surgery, during which the lesions were removed, and the frozen tissue sections were pathologically examined. Gross examination of the mass of the right breast revealed a smooth mass with a clear capsule, with the traits of a benign tumor (Fig. 2). The intraoperative histopathology revealed bilateral breast carcinoma; therefore, a bilateral mastectomy was performed and the axillary lymph nodes of each side were excised. The final pathological report revealed an invasive $2 \times 3-\mathrm{cm}^{2}$ lobular carcinoma of the left breast, without metastasis to the axillary lymph nodes (0/14). According to the American Society of Clinical Oncology/College of 
A

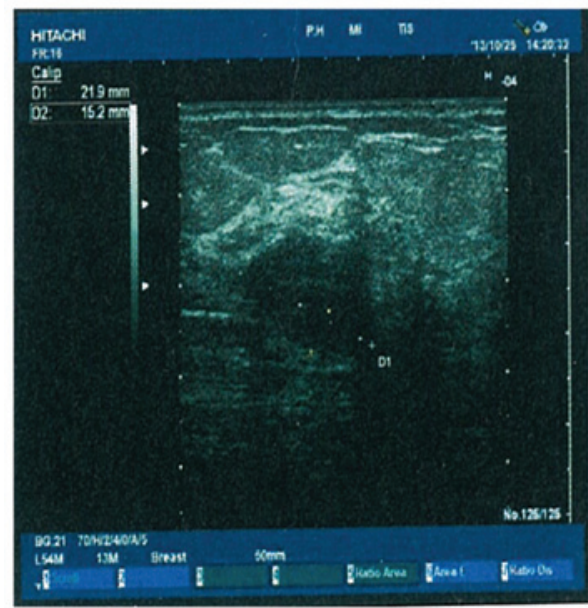

B

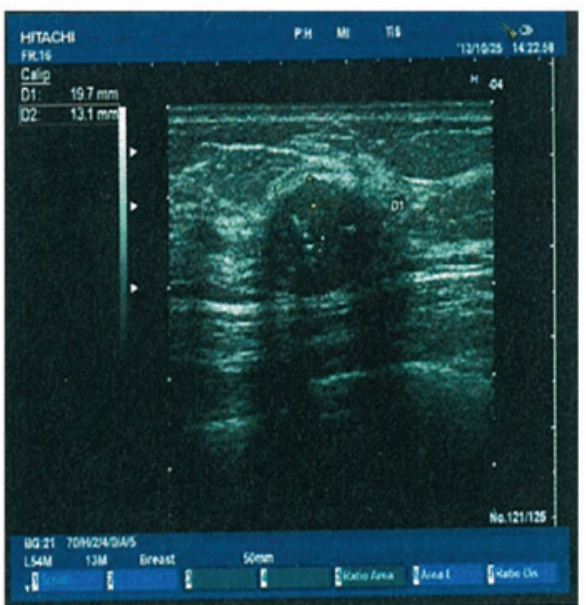

Figure 1. Breast ultrasonography showing the presence of masses within (A) the left breast and (B) the right breast.
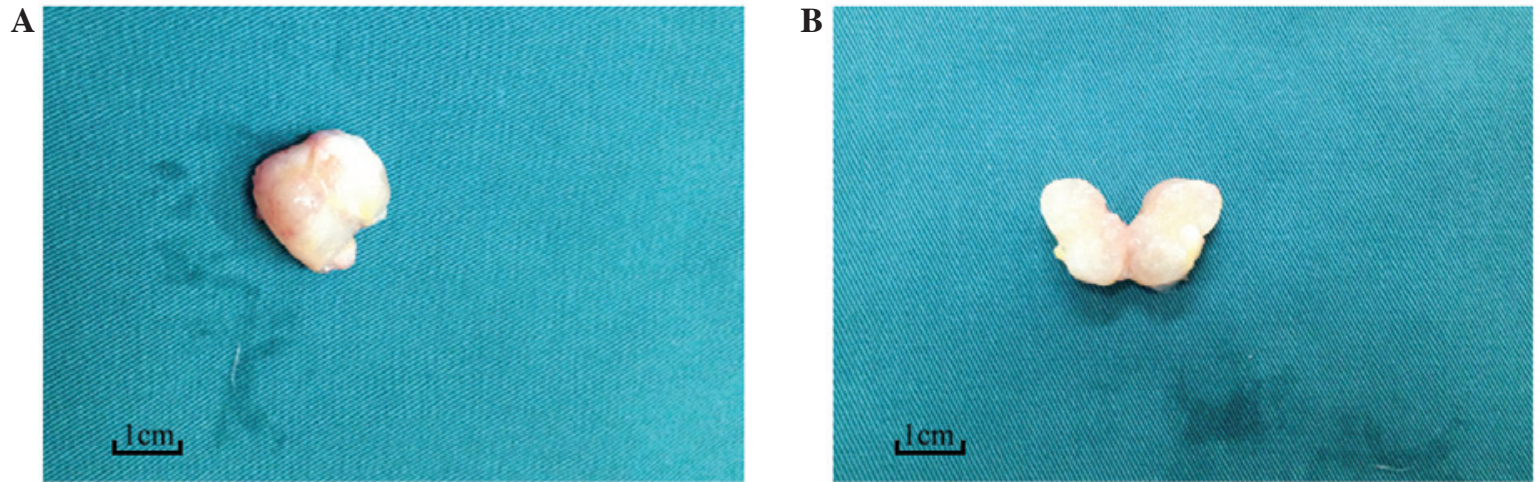

Figure 2. Gross examination of the right-sided breast mass. (A) Gross appearance, a smooth mass with a clear capsule. (B) Cut surface, bulging with a whorled appearance.

American Pathologists Guideline Recommendations for Immunohistochemical Testing $(13,14)$, immunohistochemical assay of the tumor in the left breast revealed the following: Estrogen receptor (ER)(-), progesterone receptor (PR)(-), human epidermal growth factor receptor 2 (HER2)(3+) and a Ki-67 labeling index (LI) of $60 \%$. However, a $1.5 \times 2-\mathrm{cm}^{2}$ carcinoma was present within a fibroadenoma on the right breast (mixed with invasive ductal carcinoma, grade II, without metastasis to the axillary lymph nodes) (0/16). Microscopically, the elements of fibroadenoma and carcinoma could be observed in the pathological tissue slides (Fig. 3). A second immunohistochemical assay of the tumor in the right breast showed the following: $\mathrm{ER}(+), \mathrm{PR}(+)$, HER2(+) and a Ki-67 LI of 50\%. The patient received 4 cycles of chemotherapy (epirubicin, $60 \mathrm{mg} / \mathrm{m}^{2}$ and cyclophosphamide, $600 \mathrm{mg} / \mathrm{m}^{2}$ ) every 21 days and then following four cycles chemotherapy of docetaxel $\left(100 \mathrm{mg} / \mathrm{m}^{2}\right)$ every 21 days. The patient then received endocrine therapy (tamoxifen, $20 \mathrm{mg}$, twice daily) as maintenance for 19 months. The patient had follow-up reviews once every 3 months, and has not exhibited any signs of recurrence up to the latest follow-up appointment on May 10, 2015.

\section{Discussion}

Carcinoma arising within a fibroadenoma is a rare and unique subtype of mammary cancer, which was reported by
Buzanowski-Konakry et al in 1975 (15). To date, <130 cases have been reported in the literature (16-19).

According to the known diagnostic criteria, the present case conforms to the diagnosis of sychronous bilateral primary breast cancer. However, the case is particularly unusual, as unilateral breast invasive ductal carcinoma arising within a fibroadenoma also occurred. Compared with the cases reported in the abovementioned literature that included carcinoma in situ and invasive lobular carcinoma arising within a fibroadenoma $(9,15,16)$, the current case presented invasive ductal carcinoma arising within a fibroadenoma with contralateral invasive lobular carcinoma. To the best of our knowledge, no such case has been reported previously in the literature. In the present case, two mutually separated cancerous nodes existed in the bilateral breast, and each possessed different pathological traits. While the tumor on the left was an invasive lobular carcinoma, the tumor on the right was a carcinoma within a fibroadenoma mixed with invasive ductal carcinoma. Gross examination revealed that the mass on the right possessed most of the traits of a benign tumor, which was misdiagnosed as fibroadenoma by a clinical doctor. Therefore, the breast masses could not be identified by physical and gross examinations alone. Further imaging tests and an excision were necessary to obtain a clear diagnosis. The patient was administered routine therapy regimens, which is the same treatment method as that used for the other breast cancer 
A

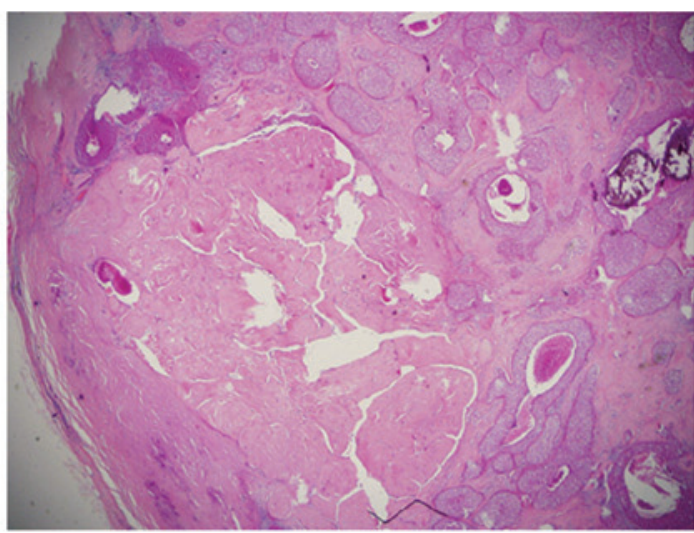

C

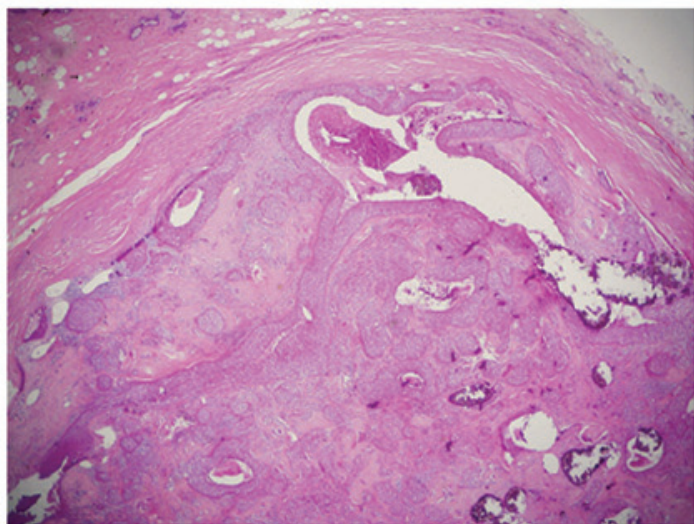

E

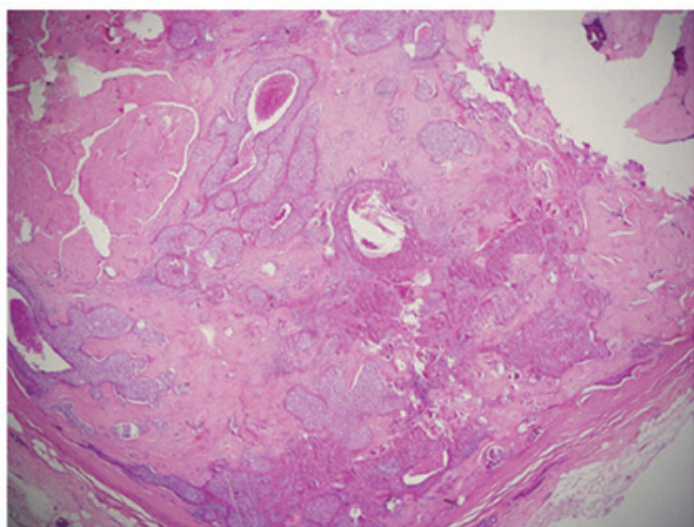

B

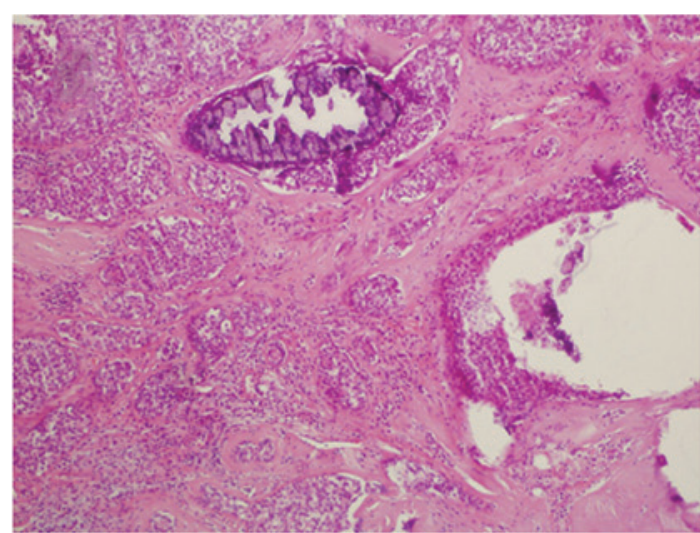

D

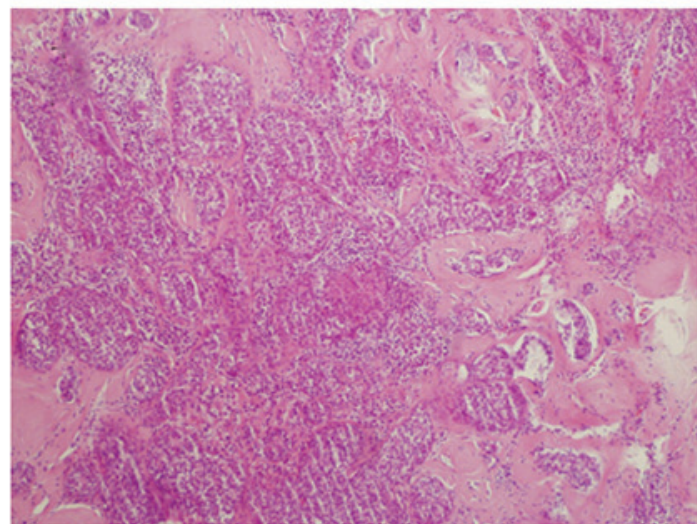

F

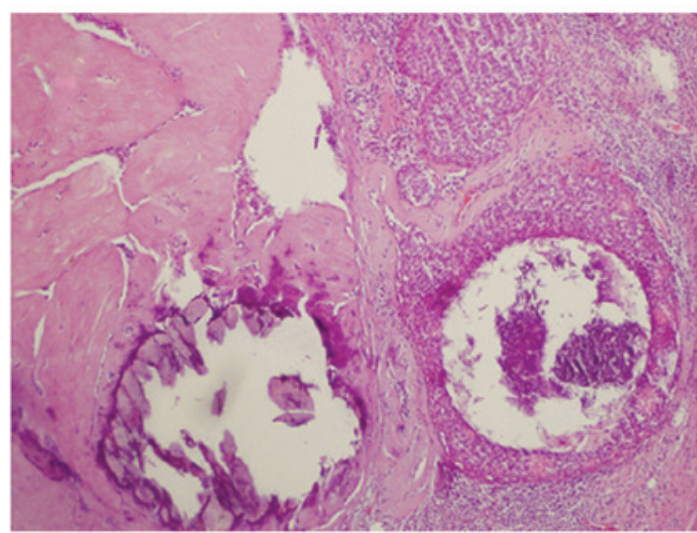

Figure 3. Microscopic examination of the carcinoma within the fibroadenoma. (A) Invasive ductal carcinoma with focal comedo necrosis and fibroadenoma (magnification, x 100). (B) Infiltrating islands of malignant ductal epithelial cells and fibroadenoma (magnification, x200). (C) Fibrous connective tissue capsule and carcinoma (magnification, x100). (D) Infiltrative ductal carcinoma and inflammatory cells in the stroma (magnification, $\mathrm{x} 200$ ). (E) Widespread and dense invasive ductal carcinoma with numerous calcified bodies overlapping between fibroadenoma and invasive ductal carcinoma (magnification, $\mathrm{x} 100$ ). (F) Cancer cell nests, composed of invasive ductal carcinoma, evolved from fibroadenoma (magnification, x200).

patients. Few studies have reported the prognosis of this type of breast cancer (20). The present patient has been closely followed up for 19 months and has not exhibited any signs of recurrence.

There are no rules guiding the therapy of a carcinoma within a fibroadenoma. Thus, it is essential to accumulate further case studies like the present study in order to be able to treat such cases with evidence-based medicine.

\section{Acknowledgements}

The current study was supported by grants from the Key Project of Health and Family Planning Commission of Hubei Province, China (grant no. WJ2015MA016) and the Training Plan of Young and Middle-Aged Backbone Talents of Medicine of Health and Family Planning Commission of Wuhan, Hubei Province, China (March, 2014).

\section{References}

1. Torre LA, Bray F, Siegel RL, et al: Global cancer statistics, 2012. CA Cancer J Clin 65: 87-108, 2015.

2. Youlden DR, Cramb SM, Dunn NA, et al: The descriptive epidemiology of female breast cancer: An international comparison of screening, incidence, survival and mortality. Cancer Epidemiol 36: 237-248, 2012.

3. Hurvitz SA, Hu Y, O'Brien N and Finn RS: Current approaches and future directions in the treatment of HER2-positive breast cancer. Cancer Treat Rev 39: 219-229, 2013.

4. Limite G, Esposito E, Sollazzo V, Ciancia G, Formisano C, Di Micco R, De Rosa D and Forestieri P: Lobular intraepithelial neoplasia arising within breast fibroadenoma. BMC Res Notes 6: 267,2013 
5. Stafyla V, Kotsifopoulos N, Grigoriades K, Kassaras G and Sakorafas GH: Lobular carcinoma in situ of the breast within a fibroadenoma. A case report. Gynecol Oncol 94: 572-574, 2004.

6. Ooe A, Takahara S, Sumiyoshi K, Yamamoto H, Shiba E and Kawai J: Preoperative diagnosis of ductal carcinoma in situ arising within a mammary fibroadenoma: A case report. Jpn J Clin Oncol 41: 918-923, 2011.

7. Hayes BD and Quinn CM. Microinvasive lobular carcinoma arising in a fibroadenoma. Int J Surg Pathol 21: 419-421, 2013.

8. Monsefi N, Nikpour H, Safavi M, Lashkarizadeh MR and Dabiri S: Mucinous subtype of invasive ductal carcinoma arising within a fibroadenoma. Arch Iran Med 16: 366-368, 2013.

9. Pacchiarotti A, Selman H, Gentile V, Pacchiarotti A, Milazzo GN, Lanzilotti G, Lofino S and Frati P: First case of transformation for breast fibroadenoma to high-grade malignant phyllodes tumor in an in vitro fertilization patient: Misdiagnosis of recurrence, treatment and review of the literature. Eur Rev Med Pharmacol Sci 17: 2495-2498, 2013

10. Nio Y, Iguchi C, Tsuboi K and Maruyama R: Ductal carcinoma in situ arising within a benign phyllodes tumor: A case report with a review of the literature. Oncol Lett 2: 223-228, 2011.

11. Smith GE and Burrows P: Ultrasound diagnosis of fibroadenomais biopsy always necessary? Clin Radiol 63: 511-515; discussion 516-517, 2008.

12. Wilkinson S and Forrest AP: Fibro-adenoma of the breast. Br J Surg 72: 838-840, 1985.

13. Hammond ME, Hayes DF, Dowsett M, et al: American Society of Clinical Oncology/College Of American Pathologists guideline recommendations for immunohistochemical testing of estrogen and progesterone receptors in breast cancer. J Clin Oncol 28: 2784-2795, 2010.
14. Lambein K, Van Bockstal M, Denys H and Libbrecht L: 2013 update of the American Society of Clinical Oncology/College of American Pathologists guideline for human epidermal growth factor receptor 2 testing: Impact on immunohistochemistry-negative breast cancers. J Clin Oncol 32: 1856-1857, 2014.

15. Buzanowski-Konakry K, Harrison EG Jr and Payne WS: Lobular carcinoma arising in fibroadenoma of the breast. Cancer 35: 450-456, 1975.

16. Diaz NM, Palmer JO and McDivitt RW: Carcinoma arising within fibroadenomas of the breast. A clinicopathologic study of 105 patients. Am J Clin Pathol 95: 614-622, 1991.

17. Fukuda M, Nagao K, Nishimura R, Matsuda M, Baba K, Ueno Y, Morinaga H, Omachi $\mathrm{H}$ and Hamada T: Carcinoma arising in fibroadenoma of the breast-a case report and review of the literature. Jpn J Surg 19: 593-596, 1989.

18. Yoshida Y, Takaoka M and Fukumoto M. Carcinoma arising in fibroadenoma: Case report and review of the world literature. J Surg Oncol 29: 132-40, 1985.

19. Tanaka A, Hirano M, Sakai Y, Ichikawa T, Nitta N and Henmy K: Fibroadenoma, mastopathy, intraductal papilloma: Relationship to carcinoma-a case report of carcinoma of the breast arising in fibroadenoma. Nihon Geka Hokan 52: 232-243, 1983 (In Japanese).

20. Gashi-Luci LH, Limani RA and Kurshumliu FI: Invasive ductal carcinoma within fibroadenoma: A case report. Cases J 2: 174, 2009. 